\title{
COUPLING AND FEEDBACK EFFECTS IN EXCITABLE SYSTEMS: ANTICIPATED SYNCHRONIZATION
}

\author{
MARZENA CISZAK*,‡, RAÚL TORAL ${ }^{*, \dagger}$ and CLAUDIO MIRASSO* \\ *Departament de Física, Universitat de les Illes Balears, \\ E-07122 Palma de Mallorca, Spain \\ $\dagger$ Instituto Mediterraneo de Estudios Avanzados, CSIC-UIB \\ E-07122 Palma de Mallorca, Spain \\ ¥marzena@imedea.uib.es
}

Received 15 October 2004

\begin{abstract}
This paper reviews our recent work on the synchronization of excitable systems in a master-slave configuration and when the slave system includes a delayed self-coupling term. Particularly, we address the existence of the so-called anticipated synchronization, i.e. a dynamical regime in which the slave system is able to reproduce in advance the evolution of the master. This is most remarkable since the anticipated synchronization appears even when the excitable spikes are induced by random terms, such as white noise. After providing a short review of the general theory of synchronization as well as the main features of excitable systems, we present numerical and experimental results in coupled excitable systems of the FitzHugh-Nagumo type driven by different types of noise. The experiments have been done in electronic implementations of the model equations. We present the conditions (values of the coupling intensity and delay time) for which the anticipated synchronization regime is a stable one and show that it is possible to increase the anticipation time by using a cascade of several coupled systems. We use a particular limit of the FitzHugh-Nagumo system, as well as a simple excitable model, to give evidence that the physical reason for the existence of anticipated synchronization is the lowering of the excitability threshold of the slave due to the coupling. Finally, we propose a hypothesis for a possible explanation of the zero-lag synchronization observed in some real neuron systems.
\end{abstract}

Keywords: Excitable systems; anticipated synchronization; cascade; zero-lag synchronization.

\section{Introduction}

\subsection{Synchronization phenomenon}

Interactions between the constituents of physical or biological systems occur due to the existence of different types of connections: global, local, unidirectional, multidirectional or others. Coupled interacting systems have been the subject of deep observation since the 17th century. This interest has led to mathematical theories which enable the understanding (at least partially) of the behavior of many coupled systems including very complex ones in wide areas of natural and technical sciences. 
Under particular conditions, coupled systems may exhibit coherent behavior. Such a phenomenon is called a synchronization and it describes a situation when several identical objects performing initially, in the absence of interactions, oscillations or rotational motions with different frequencies may, even in the presence of very weak interactions, start moving with the same or multiple frequencies. ${ }^{1}$ Typical examples of synchronized behavior in the biological systems are the coincident pulses of light produced by male fireflies or the synchronized sounds of crickets. $^{2}$ In these examples, the interactions between the insects are mainly through mutual perception, although they are also determined by environmental external stimuli. Some experiments showed that an external periodic stimulus can influence the degree and quality of the synchronization in fireflies. Another biological example is that of cardiac cells whose global synchronized activity results in regular heartbeating.

The first recorded observation of synchronization in physical systems was made by Christian Huygens in the 17th century. In order to improve the time accuracy in long sea trips, he came up with the idea of using two pendulum clocks hanging from the same rack. To his surprise, he noticed that the two pendula were beating in synchrony. This synchrony was due to the small coupling through the common frame supporting both clocks. Some advances were made in the next two centuries, when the study of synchronization was developed by E. Appleton, B. van der Pol, A. A. Andronov and A. A. Vitt. ${ }^{3,4}$ They developed a synchronization theory of electric and electromagnetic oscillations in electronics and radio-physics. Further contributions by other scientists concerned synchronization in many types of nonlinear oscillators: relaxation and forced ones, as well as in oscillators driven by noise. ${ }^{5}$

In the late 1980s researchers turned their attention to the synchronization of chaotic systems. This interest arose from the complex but at the same time deterministic characteristics of chaotic systems. Some studies were motivated by the possible applications of chaos in secure communication systems. More precisely, it was suggested the possibility of hiding a message in a chaotic signal during a transmission. ${ }^{6}$ The advantage of this type of transmission lies in the difficulty of the separation of the message from the chaotic signal with the correct receiver. If an observer knows neither the equations of motion which were used to generate the chaotic signal carrying the message nor the initial conditions, then the observer will not be able to extract the hidden message. Nevertheless, it has to be said that there have been some suggestions for methods for decryption and the usefulness of such an encryption method is still under investigation. Pioneering work on synchronous coupled chaotic systems was made by Yamada and Fujisaka in 1983, Afraimovich, Verichev and Rabinovich in 1986, and Pecora and Carroll in 1990. ${ }^{7-10}$

As was mentioned above, the interest in the synchronization of chaotic systems arises from their complex, unpredictable, but nevertheless deterministic dynamics. Other types of systems which exhibit very complex and unpredictable dynamics are stochastic systems, which are non-autonomous since they include noise in an 
additive or multiplicative (parametric) form. ${ }^{11}$ Contrary to the chaotic systems, the stochastic ones exhibit unpredictable dynamics even for short periods. Many biological systems operate in a stochastic regime, for example, neurons in the brain, cardiorespiratory systems and population systems. ${ }^{12-14}$ For that reason, a great deal of interest has been given to the synchronization of stochastic systems. ${ }^{15}$

In many cases of interest it is essential to include in the model, feedback loops which inject a delayed signal back to the system. ${ }^{16}$ These dynamical terms are generically known as time-delayed self-interactions. Delayed feedback appears in models of nonlinear optical resonators, the ocean-atmosphere system or the neuronal activity in the brain. Systems with delayed feedback become very complex to study since the effective number of degrees of freedom are very large. This can be easily understood by observing that the initial condition of a delayed differential equation is a whole interval $\left[x\left(t_{0}-\tau\right), x\left(t_{0}\right)\right]$ of function values. ${ }^{17,18}$ Thus, delayed feedback, even in linear systems, can lead to very complicated dynamics.

Acting together, coupling and delayed feedback can lead to a phenomenon called anticipated synchronization. This was first reported by Voss in $2000 .{ }^{19}$ Anticipated synchronization can occur in two unidirectionally coupled systems, when one of them (the so-called slave, $\mathbf{y}$ ) becomes synchronized with the output of the other (the master, $\mathbf{x}$ ), but its signal is shifted back in time such that $\mathbf{y}(t)=\mathbf{x}(t+\tau)$, i.e. the slave has the dynamics which the master system will have a time $\tau$ later. There are two known delayed coupling schemes which can lead to anticipated synchronization. One of them is the so-called complete replacement:

$$
\begin{aligned}
\dot{\mathbf{x}}(t) & =\mathbf{f}(\mathbf{x}(t), \mathbf{x}(t-\tau)) \\
\dot{\mathbf{y}}(t) & =\mathbf{f}(\mathbf{y}(t), \mathbf{x}(t)),
\end{aligned}
$$

where $\mathbf{x}$ and $\mathbf{y}$ are dynamical variables, $\mathbf{f}$ is a vector function and $\tau$ is a constant delay time. In Eq. (1) the time-delayed variable is replaced by a variable coming from the master system. The anticipation time can be only equal to the delay time which is present in the master system. Then the solution for the equations is $\mathbf{y}(t)=\mathbf{x}(t+\tau)$. The other scheme which, contrary to the above case, permits to obtain different anticipation times, is that with dissipative (diffusive) coupling:

$$
\begin{aligned}
\dot{\mathbf{x}}(t) & =\mathbf{f}(\mathbf{x}(t)) \\
\dot{\mathbf{y}}(t) & =\mathbf{f}(\mathbf{y}(t))+\mathcal{K}(\mathbf{x}(t)-\mathbf{y}(t-\tau)),
\end{aligned}
$$

where $\mathbf{x}$ and $\mathbf{y}$ are dynamical variables, $\mathbf{f}$ is a vector function, $\mathcal{K}$ is a matrix representing the coupling parameter and $\tau$ is a constant delay time. In this case, a delayed term appears in the equation for the slave system.

Since its discovery by Voss, there has been a wide variety of systems in which anticipated synchronization has been observed. These systems range from the simplest linear systems to complex chaotic ones, suggesting that this phenomenon is quite general. It appears in delayed coupled linear systems, for which a full stability analysis has been provided. ${ }^{20}$ Besides the systems described by differential equations, chaotic maps with delayed diffusive coupling have also been studied. ${ }^{21}$ 
The phenomenon has been found in chaotic Rössler and Lorenz systems, which may be of some interest from a practical point of view since it could enable simultaneous prediction of the chaotic signal without involving any previous calculations. ${ }^{22}$ However, in the case of coupled chaotic systems with a dissipative delayed coupling term it is very difficult to perform a stability analysis and only limited results can be obtained using linearization or numerical methods. Anticipated synchronization was also found in the delay-induced chaotic Ikeda system which describes phase shifts in nonlinear optics. ${ }^{19}$ In this system a complete replacement scheme was used since the master contains an internal delayed feedback. It was also studied in coupled laser models. ${ }^{23}$ Interestingly, larger anticipation times can be achieved by using a chain of master-slave systems. ${ }^{24}$ Finally, anticipated synchronization has been demonstrated experimentally in electronic circuits as well as in semiconductor lasers. ${ }^{25,26}$

\subsection{Excitable systems}

Neurons are classical prototypes of excitable systems: their response to an external perturbation is highly nonlinear and depends on its magnitude and timing. If the perturbation is small the system evolves back to the steady state; but if the perturbation exceeds a certain threshold (which may be well or not well defined), the system fires a pulse-like spike (action potential). According to the intuitive definition of excitability, small perturbations near the equilibrium global attractor can cause large excursions for the solution before it returns to the equilibrium. Thus a dynamical system having a stable equilibrium is excitable if there is a large amplitude periodic pseudo-orbit passing near the equilibrium. ${ }^{27}$ Following the onset of the excitation, there is an interval time, during which another perturbation does not induce a new pulse, called refractory period.

Real neurons are complicated nonlinear systems involving a large number of variables. Nevertheless, the essential features of their excitable behavior can be captured with a much reduced description introduced by Hodgkin and Huxley. ${ }^{28}$ The model originally concerned the axon of the giant squid. Because of the stilllarge complexity of the Hodgkin-Huxley (4-dimensional) system, various simpler mathematical models, which capture the key features of the full system, have been proposed. One of the best known is the FitzHugh-Nagumo model..$^{29,30}$ The resulting 2-variable model can be described by the dimensionless system:

$$
\begin{aligned}
& \dot{x}_{1}=f\left(x_{1}\right)-x_{2}+I_{a} \\
& \dot{x}_{2}=\epsilon\left(x_{1}-b x_{2}\right),
\end{aligned}
$$

where $f\left(x_{1}\right)=x_{1}\left(a-x_{1}\right)\left(x_{1}-1\right), 0<a<1$ (for the excitable regime) and $\epsilon$ and $b$ are positive constants. Here $x_{1}$ is a fast variable called the activator which is proportional to the membrane potential $V ; x_{2}$ is a slow variable called the inhibitor. In the excitable regime and for $I_{a}=0$ the system remains in the steady state $\left(x_{1}, x_{2}\right)=(0,0)$. For $I_{a} \neq 0$ and for particular values of $I_{a}$, a limit cycle oscillation appears. 

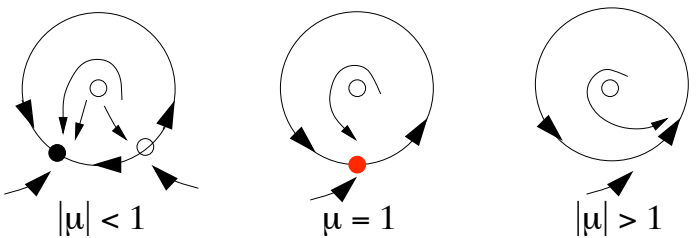

Fig. 1. Schematic presentation of the dynamics during the saddle-node bifurcation on invariant circle.

The most important features of neuron models are that they can generate regular beatings of a limit cycle nature when the applied current $I_{a}$ is within an appropriate range. There are two types of bifurcations which allow the system to be excitable and which result in a stable limit cycle when the controlled parameter changes: saddle-node bifurcation on an invariant circle (or Andronov bifurcation) and Hopf bifurcation (or Andronov-Hopf bifurcation). ${ }^{31-33}$ Saddle-node bifurcation can appear in any dimension (excitability requires dimensions equal or larger than two) and its mechanism consists of the creation and annihilation of fixed points. As a parameter of the system is varied from above to below threshold, two fixed points, one stable and one unstable move toward each other, collide, and mutually annihilate. A saddle-node bifurcation on an invariant circle appears, for example, in a model of overdamped pendulum. The dimensionless form of this model, in an overdamped limit, is the following:

$$
\dot{x}=\mu-\sin x
$$

with $x=d \theta / d \bar{t}, \bar{t}=m g L / b$ and $\mu=\Gamma / m g L$, where $m$ is a mass of pendulum, $L$ its length, $b$ is a viscous damping, $\Gamma$ is a constant applied torque and $\theta$ is an angular variable. As defined above, the parameter $\mu$ is the ratio of the applied torque to the maximum gravitational torque. Since viscosity is large, the oscillations are possible because of the applied torque - the energy is lost by damping and pumped by an applied torque. If $\mu>1$ then the applied torque can never be balanced by the gravitational torque and the pendulum will always make an excursion over an unstable fixed point $x_{+}=\arcsin \mu$ which is a saddle, and the flow consists of an oscillation of the variable $x$ (limit cycle regime). This limit cycle develops through a saddle node bifurcation on an invariant circle (Andronov) at $\mu= \pm 1$ (see Fig. 1), where the two fixed points collide and annihilate. In the latter case, if $\mu<1$, the unperturbed pendulum will never be able to reach an unstable fixed point and it will always turn back to the stable fixed point $x_{-}=\arcsin \mu$. Nevertheless, in this case the system displays an excitable behavior: if we kick the system out of its stable state with a large enough perturbation (larger than $2|\arcsin \mu|$ ), the trajectory will return to the initial state through a deterministic orbit that closely follows the heteroclinic connection of the saddle and the node [an orbit which connects two fixed points is called heteroclinic, while an orbit which connects a saddle point with itself is called homoclinic (see Fig. 2)]. It is worth noting that in a system with 


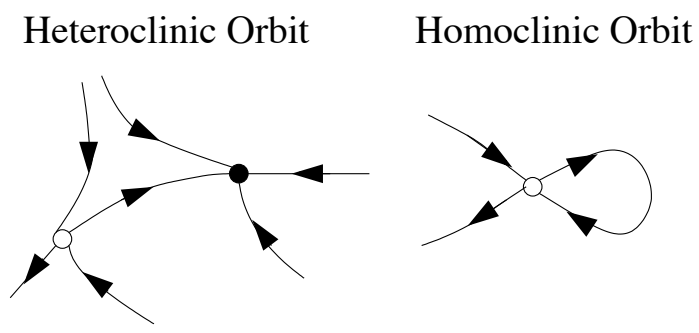

Fig. 2. Schematic presentation of the heteroclinic and homoclinic orbits.

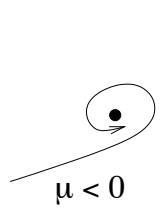

Supercritical Hopf
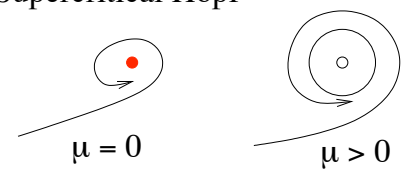

Subcritical Hopf
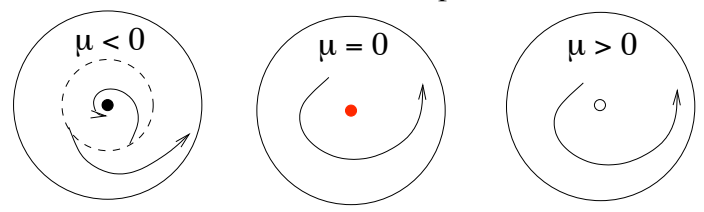

Fig. 3. Schematic presentation of the dynamics during the Hopf bifurcation.

a saddle-node bifurcation the key element to obtain excitability is the heteroclinic connection between the manifolds of the fixed points. Equation (4) appears not only in mechanics in the form of overdamped pendulum with a constant torque, but it also appears in condensed-matter physics to model dynamics of Josephson junctions, as well as in biology to model oscillating neurons, firefly flashing rhythm and human sleep-wake cycle. Usually this prototype equation carries the name Adler's equation.

Opposite to a saddle-node bifurcation, Hopf bifurcation can occur only in dimensionality two or higher and appears for example in the FitzHugh-Nagumo system. The Hopf bifurcation can be either supercritical or subcritical (see Fig. 3). A supercritical Hopf bifurcation appears when a stable spiral changes into an unstable spiral surrounded by a limit cycle and is responsible for an excitable behavior of the system. A subcritical Hopf bifurcation is responsible for bistable behavior. Before the bifurcation, the system has two attractors: a stable limit cycle and a stable fixed point at the origin. Between these two attractors lies an unstable limit cycle. At the bifurcation point ( $\mu=0$ at Fig. 3 ) the unstable limit cycle shrinks to the origin, which becomes unstable, while the stable limit cycle remains but with larger amplitude of oscillations than before the bifurcation. A definition of the Hopf bifurcation is formulated in the Hopf bifurcation theorem: a Hopf bifurcation appears if when changing some parameter of the system we observe that both eigenvalues 
(in a two-dimensional case) change from real negative to complex ones with its real parts positive. The parameter value at which the eigenvalues become complex with the vanishing real parts corresponds to the bifurcation point. ${ }^{34}$

Excitable systems were categorized by Hodgkin according to the bifurcation type into two classes. Class 1 excitable systems are the ones with a saddle-node bifurcation on an invariant circle (for example Adler's and Morris-Lecar systems). Class 2 excitable systems are characterized by the appearance of the Hopf bifurcation, as the previously described Hodgkin-Huxley and FitzHugh-Nagumo models. ${ }^{35}$ The type of bifurcation determines the neuro-computational properties of the cells. If in the system a saddle-node bifurcation occurs, the cell can fire all-or-none spikes with an arbitrary low frequency, it has a well-defined threshold manifold, and it acts as an integrator: the higher the frequency of incoming pulses, the sooner it fires. On the other hand, when a Hopf bifurcation occurs in the system, the cell fires at a certain frequency range, its spikes are not all-or-none, it does not have a well-defined threshold manifold, it can fire in response to an inhibitory pulse, and it acts as a resonator: it responds preferentially to a certain (resonant) frequency of the input. ${ }^{27}$

\section{Anticipated Synchronization in Excitable Systems Driven by Noise}

\subsection{Numerical results}

Coupled excitable systems driven by noise in the regime of anticipated synchronization were recently studied. ${ }^{36,37}$ The following scheme with diffusive coupling was considered:

$$
\begin{aligned}
\dot{\mathbf{x}}(t) & =\mathbf{f}(\mathbf{x}(t))+\mathbf{I}(t) \\
\dot{\mathbf{y}}(t) & =\mathbf{f}(\mathbf{y}(t))+\mathbf{I}(t)+\mathcal{K}[\mathbf{x}(t)-\mathbf{y}(t-\tau)],
\end{aligned}
$$

where $\mathbf{x}$ and $\mathbf{y}$ are dynamical variables, $\mathcal{K}$ is a positive defined matrix and $\mathbf{I}(t)$ represents a common external forcing. It was shown that under appropriate coupling conditions there can be a very good correlation between $\mathbf{y}(t)$ and $\mathbf{x}(t+\tau)$, even if the external forcing $I(t)$ is a noise. ${ }^{36}$

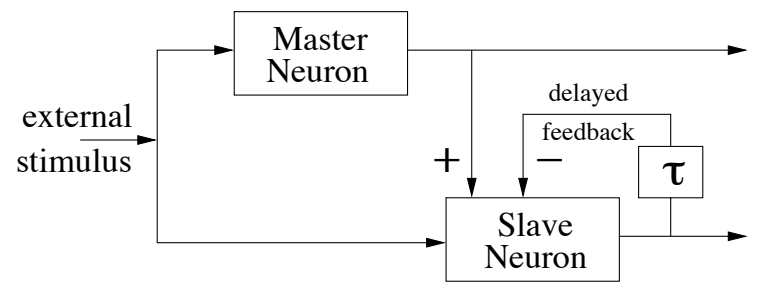

Fig. 4. Schematic diagram of two model neurons coupled in a unidirectional configuration, subjected to the same external forcing and with a feedback loop (with a delay time $\tau$ ) in the slave neuron. 
In particular, the authors studied anticipated synchronization in the FitzHughNagumo and Hodgkin-Huxley neuron models (see Sec. 1.2). By coupling two of such systems in an unidirectional configuration as in scheme (5), they found that the slave system fires the same train of spikes as the master system does, but at a certain amount of time earlier when both systems are subjected to the same external random forcing. It appears that the slave can predict the response of the master. Two FitzHugh-Nagumo systems, the master $\mathbf{x}=\left(x_{1}, x_{2}\right)$ and the slave $\mathbf{y}=\left(y_{1}, y_{2}\right)$, under unidirectional coupling are, respectively (see the schematic diagram shown in Fig. 4):

$$
\begin{aligned}
& \dot{x_{1}}=-x_{1}\left(x_{1}-a\right)\left(x_{1}-1\right)-x_{2}+I(t) \\
& \dot{x_{2}}=\epsilon\left(x_{1}-b x_{2}\right)
\end{aligned}
$$

and

$$
\begin{aligned}
& \dot{y_{1}}=-y_{1}\left(y_{1}-a\right)\left(y_{1}-1\right)-y_{2}+I(t)+K\left[x_{1}(t)-y_{1}(t-\tau)\right] \\
& \dot{y_{2}}=\epsilon\left(y_{1}-b y_{2}\right)
\end{aligned}
$$

where $a, b$, and $\epsilon$ are constants, $K$ is the positive coupling strength and $\tau$ is a delay time. Different types of random external forcing $I(t)$ (see Fig. 5) were considered. The first one, "telegraph-like noise," corresponds to a random process whose amplitude remains constant for a time $T$, switching subsequently to a new random value chosen uniformly in $\left[I_{0}-D, I_{0}+D\right]$, where $D$ is the noise intensity and $I_{0}$

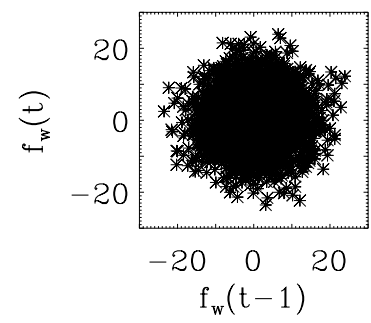

(a)

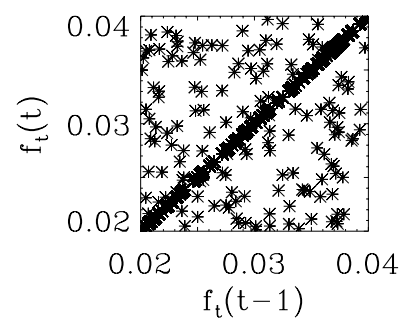

(c)

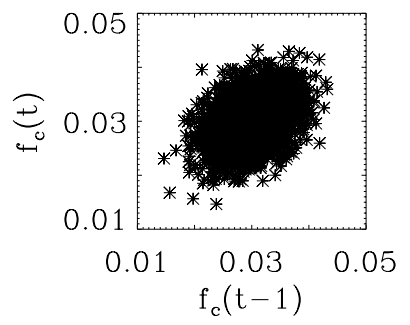

(b)

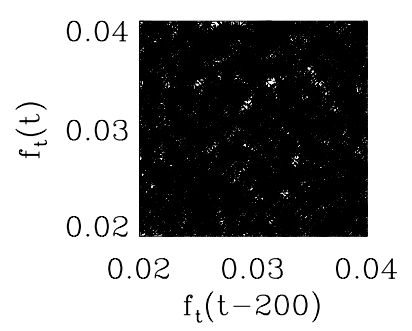

(d)

Fig. 5. Return map $f_{k}(t)$ versus $f_{k}(t-1)$ for (a) white noise $(k=w)$, (b) colored noise $(k=c)$, (c) telegraph-like noise $(k=t)$ and for $(\mathrm{d}) f_{k}(t)$ versus $f_{k}(t-200)$ for telegraph-like noise $(k=t)$. Diagrams (c) and (d) for telegraph-like noise show that it remains constant during a particular period of time. 


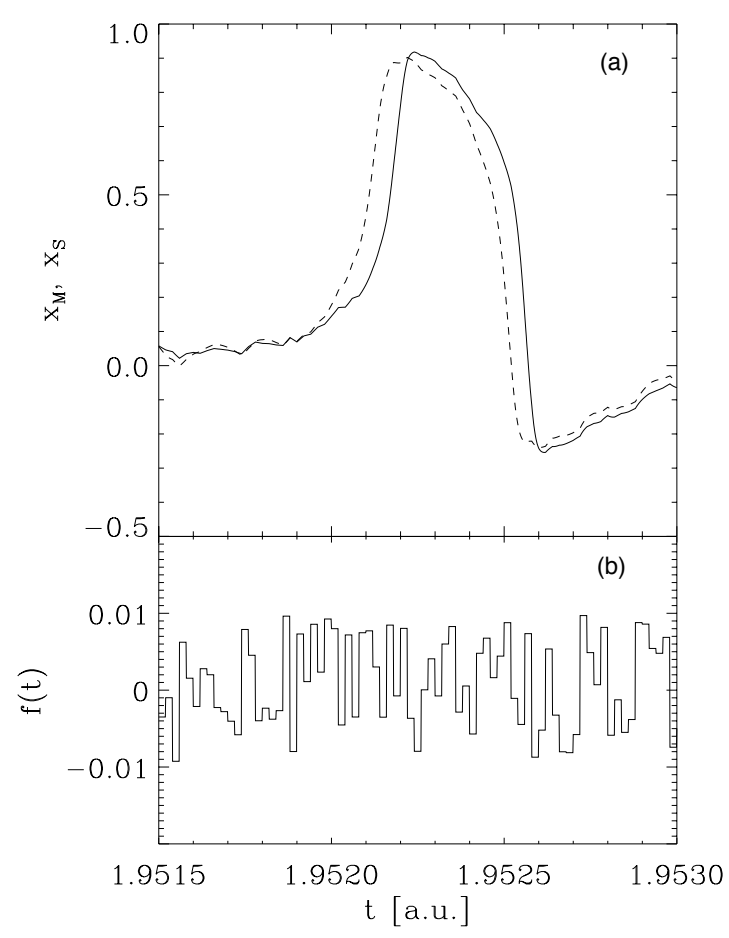

Fig. 6. Anticipated synchronization obtained from a numerical integration of the FitzHughNagumo set of Eqs. (6) and (7). The parameters are: $a=0.139, b=2.54, \epsilon=0.008, \tau=4$ and $K=0.15$. The external forcing $I(t)$ (displayed in the lower panel) is a telegraph-like noise which changes randomly its amplitude with a period $T$ (in this case $T=2$ ). Notice (upper panel) that the pulse of the slave system $y_{1}(t)$ (dashed line) anticipates the pulse of the master system $x_{1}(t)$ (solid line) by a time approximately equal to the time delay $\tau=4$.

is a constant [Figs. 5(c) and 5(d)]. If the effect of the perturbation is not strong enough the system does not fire a pulse. Moreover if the system fires a pulse it has a refractory time during which, another firing is not possible. These two features make the considered system unpredictable. Figure 6 shows that anticipation occurs with this type of random external forcing for an appropriate value of the coupling strength $K$ : after an initial transient time the two systems synchronize such that the slave system anticipates the firings of the master system by a time $\tau$. The anticipated synchronization seems to be a local process. In the absence of firings there is no anticipation (see Sec. 3 for an explanation of this observation). The same qualitative results were found with other types of external forcing such as colored and white noise. Figures $7(\mathrm{a})$ and $7(\mathrm{~b})$ display the spikes of the master and slave systems when $I(t)$ is a Gaussian white noise.

Sometimes the slave system makes an error in anticipating the master firings. While the slave system always fires a pulse when the master system fires a pulse, it might also fire an "extra" pulse, which has no corresponding pulse in the master [see Fig. 7(a)]. It was found that the longer the anticipation time $\tau$, the larger 

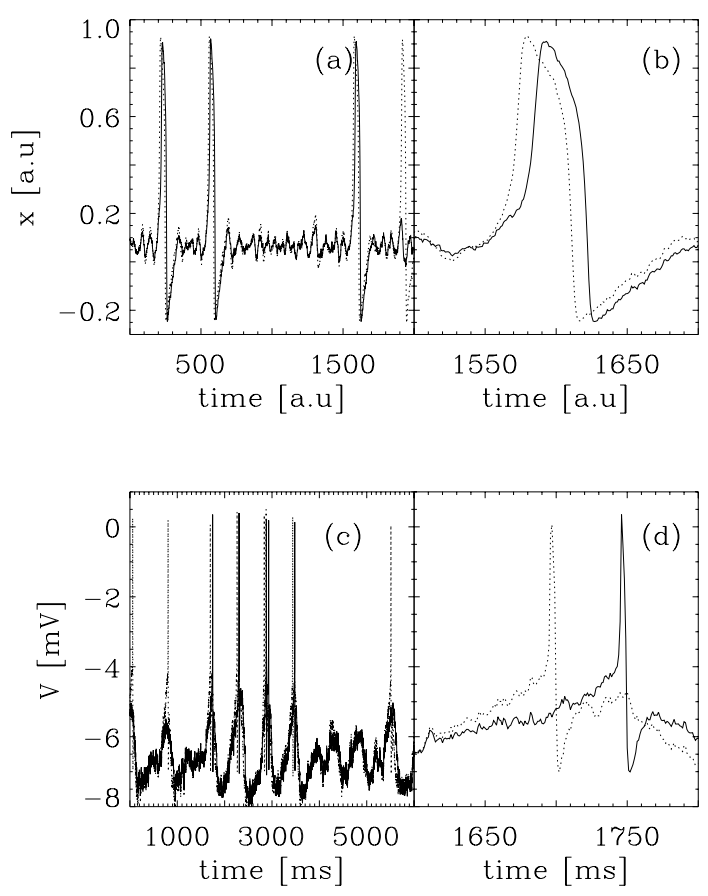

(e)

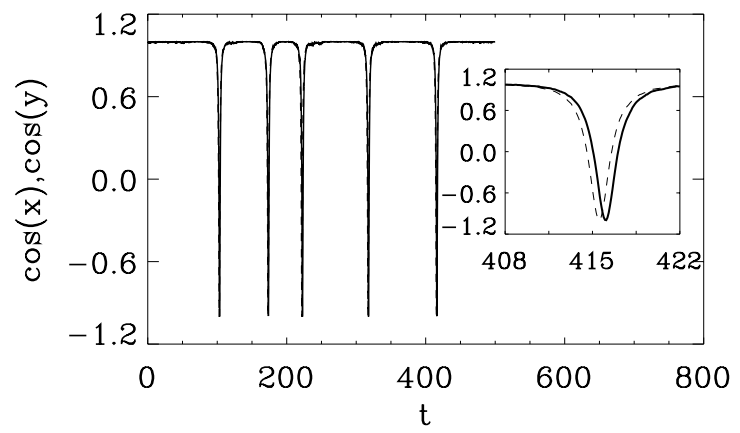

Fig. 7. Trains of spikes obtained from numerical simulations of models of unidirectionally coupled neurons subjected to the same external forcing, which is a Gaussian white noise: (a)-(b) two FitzHugh-Nagumo neurons; Eqs. (6) and (7), with the parameters in the coupling term $K=0.03$ and $\tau=10 ;$ (c)-(d) two Hodgkin-Huxley neurons with the parameters in the coupling term $K=0.03 \mathrm{~ms}^{-1}$ and $\tau=50 \mathrm{~ms}$; (e) two Adler systems, Eq. (4), with the parameters in the coupling term $K=0.01$ and $\tau=1$. Left panels show typical spike trains; right panels show with detail a single spike. The solid (dashed) line represents the output of the master (slave) system.

the number of errors and loss of anticipated synchronization occurs. However, for a given anticipation time, the number of errors can be reduced considerably if a "cascade" of an adequate number of slave neurons is considered. 


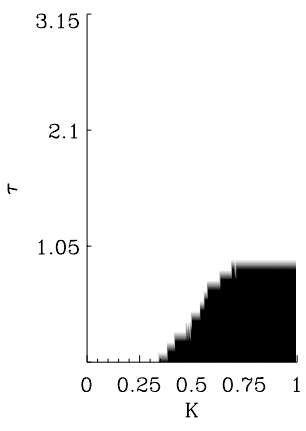

(a)

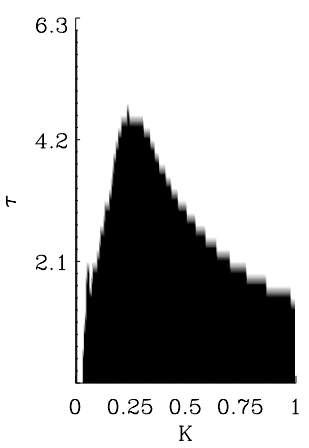

(b)

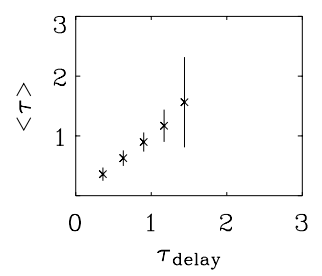

(c)

Fig. 8. Stability regime in the coupling parameter $K$ versus the delay time $\tau$ for one slave when both master and slave systems are driven by different noises. Black color represents: (a) standard deviation $\sigma_{\langle\tau\rangle}<0.16$ and (b) relative error $E_{r}<0.1$. (c) The dependence of the mean anticipation times measured at the threshold value $x_{\text {th }}=0.65$ for a coupling value $K=1$ versus the delay time used in equations (cross signs). Vertical lines correspond to the standard deviations for which the longest one corresponds to $\sigma=0.75$.

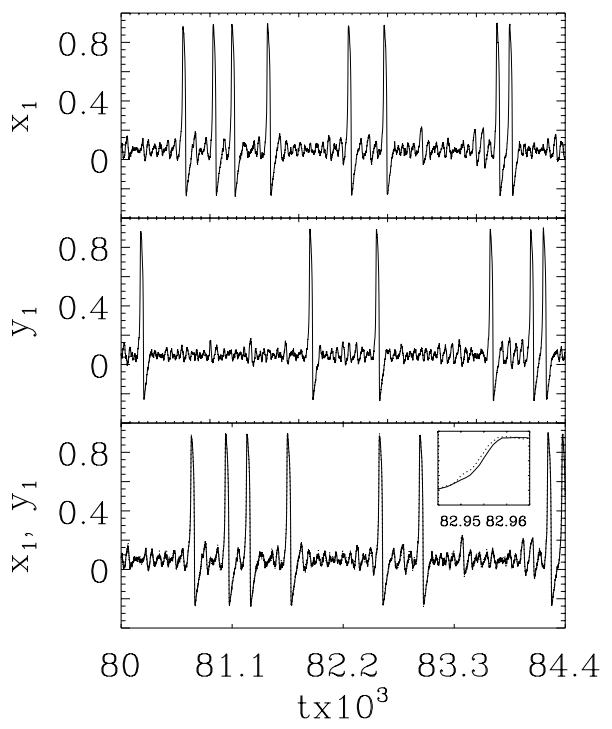

Fig. 9. Time series for the master (upper panel), uncoupled slave (middle panel) and coupled slave (lower panel) with coupling parameters $K=1$ and $\tau=1$. Both systems are driven by different realizations of white noise.

\subsection{Systems with uncommon forcing}

Numerical results (see Figs. 8 and 9) show that anticipated synchronization is possible when both master and slave systems are driven by different realizations of white noise. We obtain a smaller stability region where the maximum anticipation 
time $\tau$ is shifted in the direction of larger coupling values. This result shows that even in the presence of different white noise sources in both master and slave systems, a good anticipated synchronization can still be observed, although for larger coupling values. In an instability region, two types of errors are observed: additional spikes which occur only in the slave system and the deviation of the anticipation time when compared to the spikes from the same time series. The appearance of additional spikes is described by a relative error which is defined using the relative number of spikes in master $\left(N_{m}\right)$ and slave $\left(N_{s}\right)$ systems: $E_{r}=\left(N_{s}-N_{m}\right) / N_{m}$, while the deviation of the anticipation time is described by the standard deviation of an average anticipation time $\langle\tau\rangle: \sigma_{\langle\tau\rangle}$. The result shows that the anticipated synchronization can appear even if different noises are injected into the master and slave, which is interesting from a practical point of view. In real systems, biological ones or artificially designed ones, it is very rare to find two systems that are subject to the same noise. Thus, the robustness of this phenomenon in this case could become a useful feature.

\subsection{Experiment}

The implemented FitzHugh-Nagumo model in analog hardware was constructed with two coupled electronic neurons. The electronic neurons were built using operational amplifiers and the cubic nonlinearity described by $x(x-a)(x-1)$ was implemented (see Ref. 36 for technical details). The electronic coupled neurons behaved very similar to that in the numerical simulations. For an appropriate value of the coupling resistance $R_{C}$ (which plays the role of a coupling constant), it was observed that after a transient, the master and slave electronic neurons synchronize in such a way that the slave neuron anticipates the fires of the master neuron by a time interval approximately equal to the delay time $\tau$ of the feedback mechanism. Figure 10(a) shows a typical spike train, and Fig. 10(b) displays in detail a single spike. Without coupling and feedback $\left(R_{C}=R_{D}=0\right)$ the neurons fired pulses which were in general, unsynchronized (due to the mismatch between the circuits).

\subsection{Cascade of neurons}

In this section, we present the results of the effect of cascading several slaves units. For this purpose we assume an array of slave systems that are connected unidirectionally, as it is shown in Fig. 11, that can be described by the following set of equations:

$$
\begin{aligned}
\dot{\mathbf{x}} & =\mathbf{f}(\mathbf{x})+\mathbf{I}_{0}(t) \\
\dot{\mathbf{y}}^{1} & =\mathbf{f}\left(\mathbf{y}^{1}\right)+\mathbf{I}_{1}(t)+K\left(\mathbf{x}(t)-\mathbf{y}^{1}\left(t-\tau_{1}\right)\right) \\
\vdots & \\
\dot{\mathbf{y}}^{N} & =\mathbf{f}\left(\mathbf{y}^{N}\right)+\mathbf{I}_{N}(t)+K\left(\mathbf{y}^{N-1}(t)-\mathbf{y}^{N}\left(t-\tau_{N}\right)\right),
\end{aligned}
$$


(a)

(b)
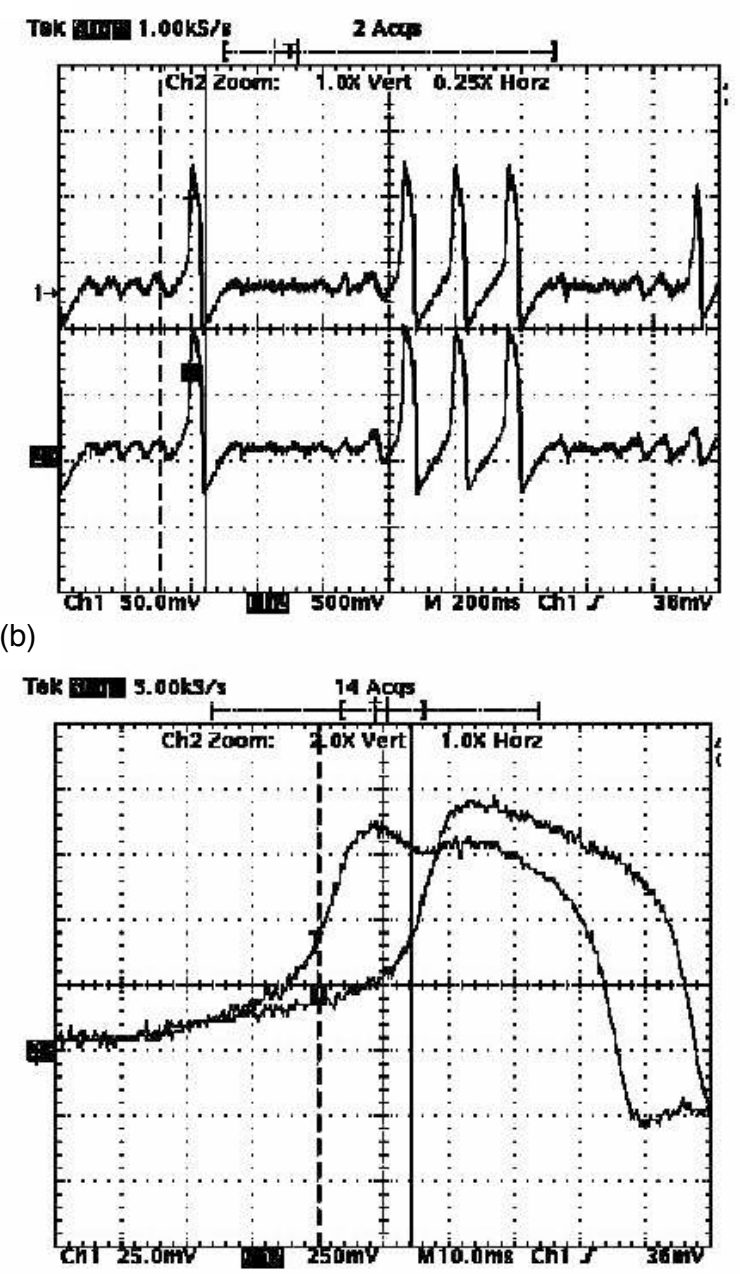

Fig. 10. (a) Experimental train of spikes that shows anticipation in the spikes fired by the slave neuron (upper trace) with respect to the spikes fired by the master neuron (lower trace). (b) Detail of a spike fired by the master neuron and anticipated spike fired by the slave neuron. The anticipation time is $14 \mathrm{~ms}$ approximately.

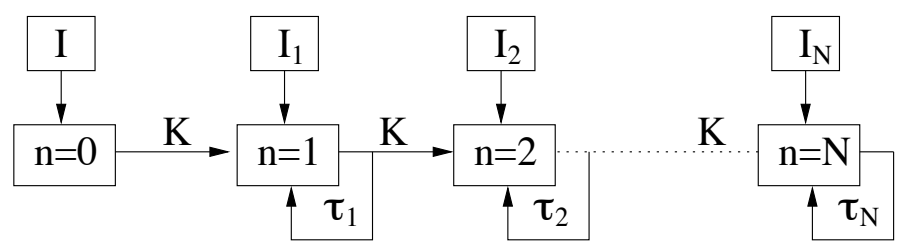

Fig. 11. An array consisting of a master system $(n=0)$ and $N$ slave systems which are fed back with a delay time $\tau$ and coupled unidirectionally with each other with a coupling parameter $K$. 

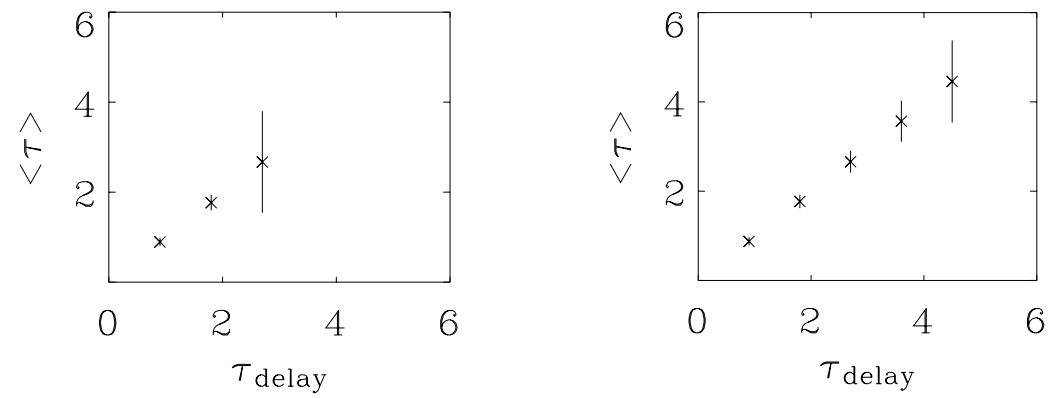

Fig. 12. Dependence of the mean anticipation time measured at the threshold value $x_{\mathrm{th}}=0.65$ for coupling value $K=0.5$ versus the delay time used in equations for the case of one slave (left plot) and three slaves with equal delays in each of them (right plot). The standard deviations are plotted in the form of vertical lines.

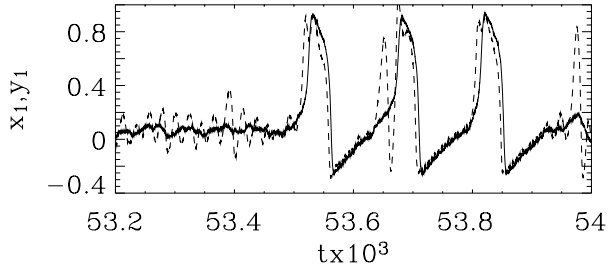

(a)

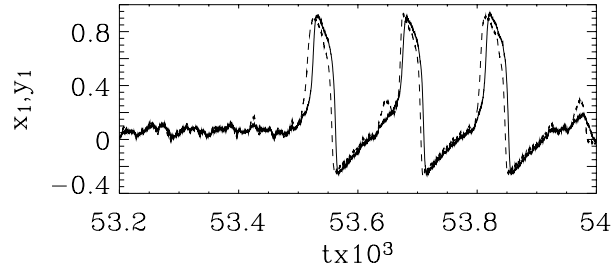

(b)

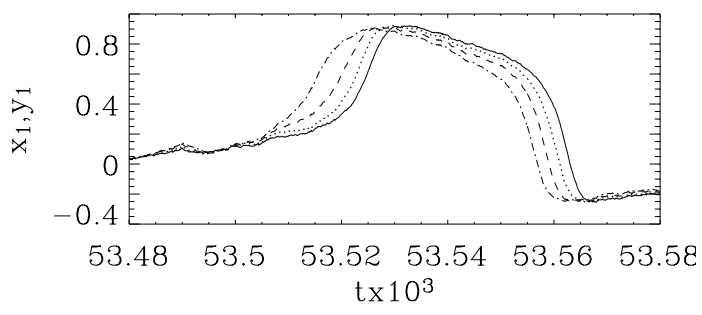

(c)

Fig. 13. (a) Time series of the master system (solid line) and first slave system (dashed line) for $\tau=6$ and $K=0.25$. (b) Time series of master system (solid line) and third slave system in a cascade (dashed line) for $\tau_{i}=2$ (where $i=1,2,3$ ) and $K=0.25$. (c) Time series of one peak of the master system (solid line), first slave (dotted line), second slave (dashed line) and third slave (dashed-dotted line).

where $N$ is the number of slave systems. The approximate possible solution for this system is:

$$
y_{1}^{N}(t-\tau)=y_{1}^{N}\left(t-\sum_{n=1}^{N} \tau_{n}\right) \approx x_{1}(t) .
$$

Numerical results shown in Fig. 12 demonstrate that cascading several slave units allows the improvement of the anticipated synchronization quality and also reduces 


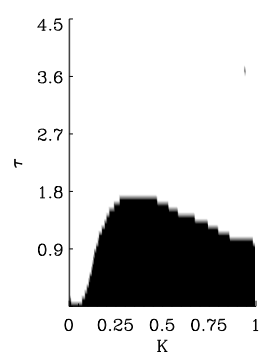

(a)

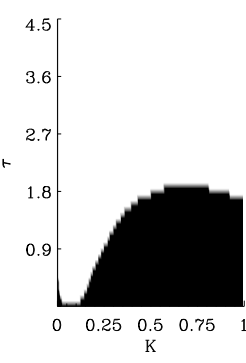

(b)

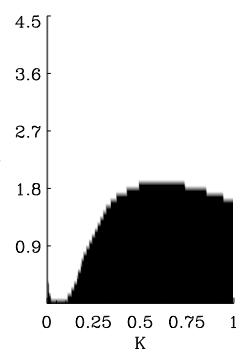

(c)

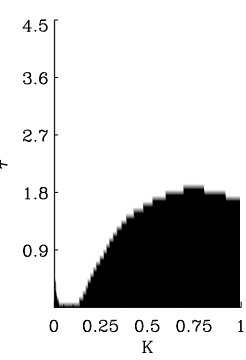

(d)

Fig. 14. Stability region estimated numerically and determined by the dependence of the coupling parameters $\tau$ and $K$ for one slave (a) and for three slaves with the following configuration of time delays: (b) $\tau_{1}=\tau_{2}=\tau_{3}=2$; (c) $\tau_{1}=1, \tau_{2}=2$ and $\tau_{3}=3$; (d) $\tau_{1}=3, \tau_{2}=2$ and $\tau_{3}=1$. Dark regions correspond to the standard deviation value $\sigma<0.15$.

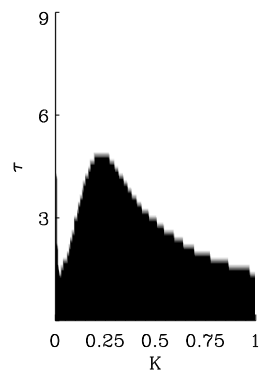

(a)

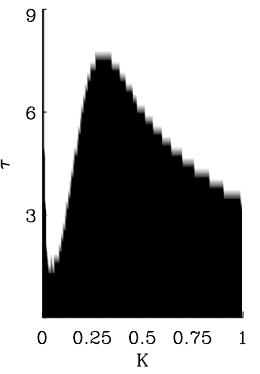

(b)

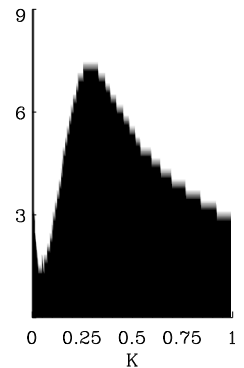

(c)

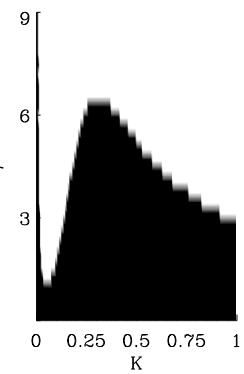

(d)

Fig. 15. Stability region in terms of the relative error $E_{r}$ for one slave (a) and a cascade of three slaves with $\tau_{i}=\tau_{j}$ for $i, j=1,2,3$; (b), $\tau_{1}<\tau_{2}<\tau_{3}$; and (c) $\tau_{1}>\tau_{2}>\tau_{3}$ (d). Black region corresponds to $E_{r}<0.1$.

errors, as it can be seen in Fig. 13. From numerical simulations of FitzHugh-Nagumo neurons with white noise (see Fig. 14), the stability region, considered in terms of standard deviation $\sigma$, changes in the following way. When $\tau_{1}<\tau_{2}<\tau_{3}$, we observe a slight shift of the maximum possible $\tau$ in the direction of smaller coupling constants $K$, in comparison with the case $\tau_{1}=\tau_{2}=\tau_{3}$. Meanwhile, for $\tau_{1}>\tau_{2}>\tau_{3}$, the maximum $\tau$ is shifted in the direction of larger coupling constants $K$, in comparison with the case $\tau_{1}=\tau_{2}=\tau_{3}$. Apart from the fact that the maximum possible anticipation time in the three types of configurations is the same, qualitatively it seems that the configuration $\tau_{1}=\tau_{2}=\tau_{3}$ is favored and has the widest stability region in the parameter space $\tau(K)$.

A larger enhancement is observed (see Fig. 15) for the relative error (the rate of additional spikes). The best configuration is again the one with equal delays in all slaves $\tau_{1}=\tau_{2}=\tau_{3}$, while configurations with different delays give smaller regions of stability. 


\section{Dynamical Mechanism of Anticipated Synchronization in Excitable Systems}

The anticipated synchronization regime has often been described as a rather counterintuitive phenomenon because of the possibility of the slave system to anticipate the unpredictable evolution of the master one. ${ }^{19,20,25}$ It was given however, a simple explanation for the physical mechanism behind the anticipated synchronization. ${ }^{38}$ The conclusion is that the anticipation in the slave is due to a reduction of its excitability threshold induced by the delayed coupling term. As a consequence, the master and the slave respond to the common external forcing with different response times. A numerical proof for the lowering of an excitability threshold in the slave system is presented in Fig. 16 on the examples of FitzHugh-Nagumo and Adler systems. Two identical systems coupled unidirectionally with a delayed coupling term are perturbed simultaneously by the $\delta$-like pulse. For large enough amplitude of perturbation, both systems fire a spike, with a slave which fires before the master does [Fig. 16(a)]. Whereas for the smaller amplitude of perturbation, there is only a spike fired by the slave [Fig. 16(b)]. Further decreasing the amplitude of the perturbation, there are no firings, neither in the master nor in the slave [Fig. 16(c)]. The proposed dynamical picture allows the explanation of all the general features of the phenomenon as well as to determine in a natural way, the maximum allowed anticipation time which can be defined as the time interval between the applied perturbation and the response time of the master system.

FitzHugh-Nagumo systems:

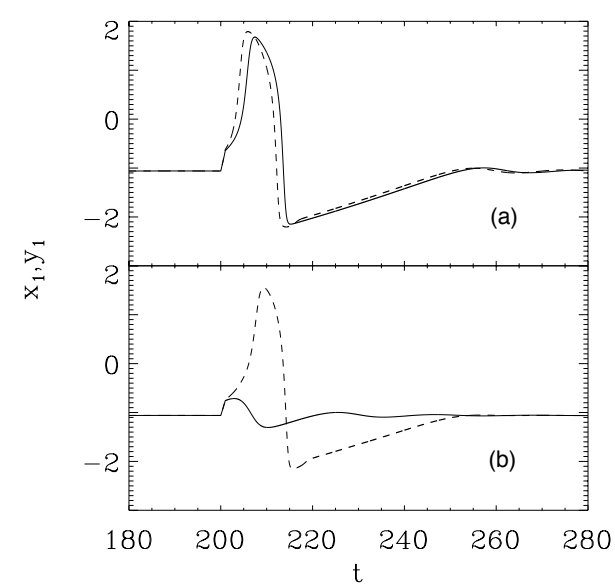

Adler systems:
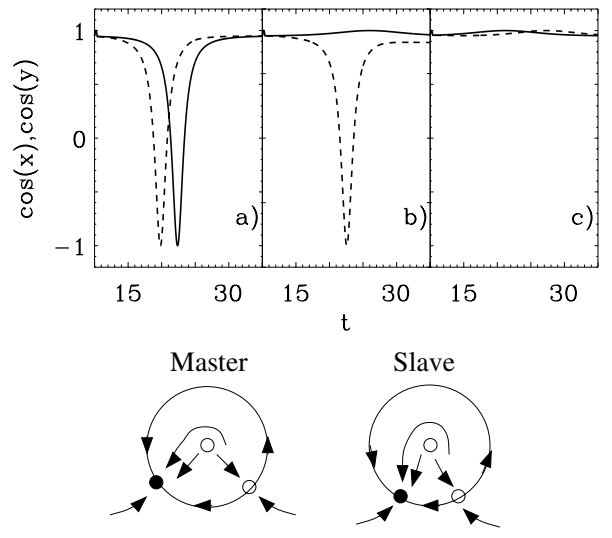

Fig. 16. Response of the master $\left(x_{1}\right.$, solid line $)$ and slave $\left(y_{1}\right.$, dashed line $)$ for two coupled FitzHugh-Nagumo and two coupled Adler systems. Parameters for the FitzHugh-Nagumo case: $a=-1.01, \epsilon=0.09, \tau=4, K=0.1$, perturbation at the time $t_{0}=200$ with duration $\Delta t=1$ and amplitude: (a) $p=0.4$ and (b) $p=0.3$. Parameters for the Adler case: $\mu=0.95, \tau=5$, $K=0.01$, perturbation at the time $t_{0}=10$ with duration $\Delta t=0.4$ and amplitude: (a) $p=1.7$, (b) $p=1.65$ and (c) $p=1.61$. Lower left panel: the graphical representation of the master and slave excitability thresholds in Adler system. 


\section{Zero-Lag Synchronization in Real Neurons}

Experiments on brain activity revealed the existence of simultaneous oscillations in the activity of cortical areas separated by several millimeters, or even located in different hemispheres, and between gamma oscillations $(30-100 \mathrm{~Hz})$ of neurons separated by millimeter distances. ${ }^{39,40}$ One explanation for these zero-lag correlations suggests that it could be simply a statistical artefact, while other explanations involve models for coexistence of doublet firing of single neurons which enable coherent oscillations. ${ }^{41,42}$ Some authors suggest that the existence of zero-lag oscillations is necessary for spatiotemporal integration of the activity of the axons. ${ }^{43}$ Several authors have pointed out that the simultaneous firing of selective neurons in the brain (so-called temporal binding) plays a crucial role in visual awareness as well as in memory structuring. ${ }^{44}$ Experimental observations which exhibit synchronized signal firings at zero-lag brought us to the speculation that in real biological systems the synchronization between neurons with different excitability thresholds may appear (see Fig. 17).

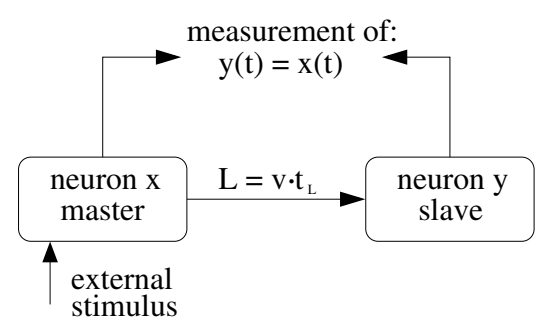

(a)

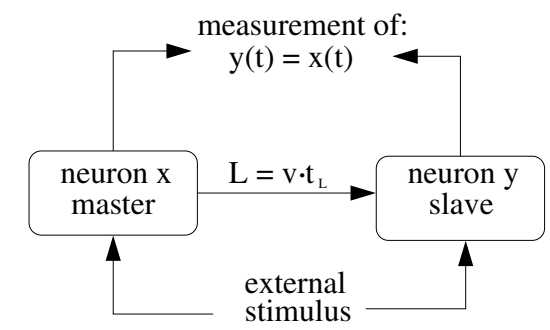

(b)

Fig. 17. Measurements of the signals coming from coupled neurons reveal zero-lag synchronization despite the fact that they are separated in space by a distance $L$. As an example we consider two cases when (a) the external stimulus is applied only to the master system or (b) the external stimulus is applied simultaneously to the master and to the slave.

We consider two cases: coupled systems where only the master is feeded with an external stimulus [Fig. 17(a)], and coupled systems which are feeded simultaneously by a common external stimulus [Fig. 17(b)]. We consider the master-slave configuration of two Adler systems:

$$
\begin{aligned}
& \dot{x}=\mu-\cos x+I_{x}(t) \\
& \dot{y}=\mu-\cos y+I_{y}(t)+K\left(x\left(t-t_{L}\right)-y(t-\tau)\right)
\end{aligned}
$$

where $t_{L}$ is the time it takes for the signal to travel from the master neuron to the slave one, and $I_{x}\left(I_{y}\right)$ are external stimuli injected into master (slave) system. Following an explanation of the mechanism of anticipated synchronization in excitable systems, ${ }^{38}$ we assume that the slave system has already an excitability threshold lower than that of the master and replace a delayed coupling term $K\left(x-y_{\tau}\right)$ with 
a synchronization coupling term $K(x-y)$. Thus we consider two coupled Adler equations with different parameter values in the master and the slave:

$$
\begin{aligned}
& \dot{x}=\mu-\cos x+I_{x}(t) \\
& \dot{y}=\mu^{\prime}-\cos y+I_{y}(t)+K\left(x\left(t-t_{L}\right)-y(t)\right) .
\end{aligned}
$$

Figures $17(\mathrm{a})$ and 17(b) correspond adequately to the cases in Eq. (11) when $I_{x} \neq$ 0 and $I_{y}=0$, and when both stimuli in the master and slave are nonzero and equal, i.e. $I_{x}=I_{y}$. We have studied in more detail the case when master and slave systems have different parameter values, since Eq. (11) can be considered in some approximation as a consequence of Eq. (10). However, it is worth noting that including a delayed feedback loop in the slave system is more advantageous since it enables the control of the height of the excitability threshold, resulting in the ability to control the magnitude of the response time. This is especially important when thinking about the real neuronal systems in which the response plasticity due to the external stimuli were observed experimentally. ${ }^{45}$ Numerical simulations have shown (see Fig. 18) that in the case of Eq. (11), for $I_{x}=I_{y}, t_{L}=0$ and particular value of $\delta \mu=\mu^{\prime}-\mu$, anticipation or retardation of the slave's pulse due to the master pulse is observed. Thus the compensation of the time $t_{L}$ needed for an electric pulse to travel from the master to the slave neuron could be achieved if $t_{\text {ant }}=t_{L}$. Note however that in this case even in the absence of the coupling term (or $K=0$ ), both neurons would be able to synchronize with zero-lag by a common forcing. Conversely, in the previous case, if the stimulus in the slave is absent, $I_{y}=0$, the coupling parameter $K$ plays a crucial role in the synchronization of both master and slave systems. Numerical simulations of Eq. (11) with $I_{y}=0$ and $t_{L}=0$ show also that in this case, anticipation is possible. Thus we can observe $y(t)=x(t)$ for $t_{L} \neq 0$ if the difference between the response time of the master neuron $t_{r}^{m}$ and the response time of the slave neuron $t_{r}^{s}$ corresponds exactly to the time needed for an electric pulse to travel from the master to the slave neuron $t_{L}=t_{r}^{m}-t_{r}^{s}$. Then the time it takes for the pulse to travel through the axon could be compensated, giving

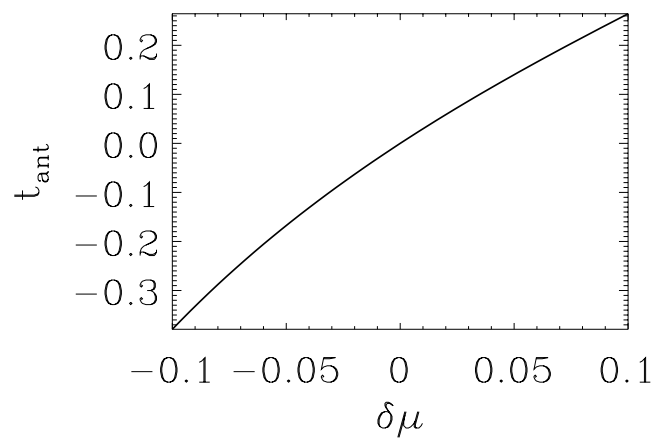

Fig. 18. Dependence of the response time of a slave Adler system on the parameter's difference $\delta \mu$ between master and slave. 


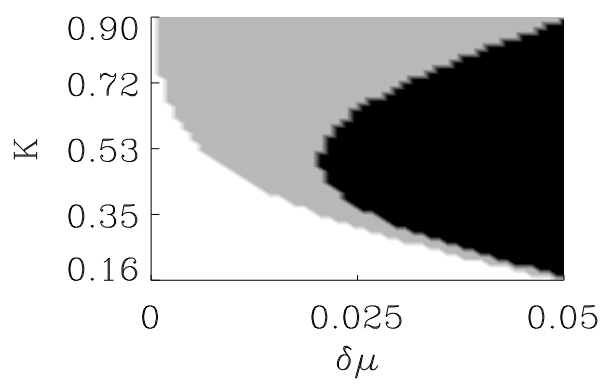

Fig. 19. Appearance of anticipated and retarded response of a slave in Adler system, depending on the magnitudes of coupling parameter $K$ and difference $\delta \mu=\mu^{\prime}-\mu$ between the parameters in the master and slave systems with $\mu=0.95$ in the master. The white region corresponds to: $t_{r}^{m}-t_{r}^{s}<0$ (the slave responds later than the master), grey: $0.25>t_{r}^{m}-t_{r}^{s}>0$; and black: $t_{r}^{m}-t_{r}^{s}>0.25$ (in the two last cases the slave responds faster than the master); where $t_{r}^{m}\left(t_{r}^{s}\right)$ is the response time of the master (slave).

rise to a simultaneous firing of neurons. In Fig. 19 we present numerical results for the appearance of retardation and anticipation of the slave in the parameter space $K$ and $\delta \mu$.

\section{Conclusions}

In this review we have presented results on delayed coupled equations for excitable systems driven by a common and even uncommon external forcing, which under appropriate conditions may lead to anticipated synchronization. This happens despite the fact that the anticipated synchronization manifold is not a solution of the equations. The FitzHugh-Nagumo model was also implemented in analog hardware, showing that the anticipation phenomenon is very general and robust.

Two types of errors appearing only in the slave system have been observed, relative error and standard deviation of anticipation times, have been revealed to be proportional only for small delay times, and deviations of anticipation times appeared more often, even in the absence of additional firings. It was also shown that the anticipated synchronization can be improved by cascading several units of slave systems. The appearance of additional firings in the slave system and the fact that the response time of an excitable system depends on its excitability threshold (which is determined by the parameters of the system), lead to the explanation of the anticipated synchronization in excitable systems in terms of a threshold reduction.

Finally, we have proposed a hypothesis that the phenomenon of anticipated synchronization might be responsible for the experimental observations of the zerolag synchronization between spatially separated coupled neurons.

\section{Acknowledgment}

R. Toral and C. Mirasso acknowledge the project CONOCE2: FIS2004-00953. 


\section{References}

1. I. I. Blekhman, Synchronization in Science and Technology (Asme Press, New York, 1988).

2. S. T. Strogatz, Nonlinear Dynamics and Chaos: With Applications to Physics, Biology, Chemistry and Engineering (Addison-Wesley, 1997).

3. E. V. Appleton, Proc. Cambridge Phil. Soc. (Math. Phys. Sci.) 21 (1922) 231.

4. B. van der Pol, Phil. Mag. 3 (1927) 64; A. A. Andronov and A. A. Vitt, Zhurnal Prikladnoy Fisiki 7 (1934) 4.

5. A. Pikovsky, M. Rosenblum and J. Kurths, Int. J. Bifurc. Chaos 10 (2000) 2291.

6. K. M. Cuomo and A. V. Oppenheim, Phys. Rev. Lett. 71 (1993) 65.

7. T. Yamada and H. Fujisaka, Prog. Theor. Phys. 70 (1983) 1240.

8. T. Yamada and H. Fujisaka, Prog. Theor. Phys. 72 (1984) 885.

9. V. S. Afraimovich, N. N. Verichev and M. I. Rabinovich, Inv. VUZ Rasiofiz. RPQAEC 29 (1986) 795.

10. L. M. Pecora and T. L. Carroll, Phys. Rev. Lett. 64 (1990) 821.

11. W. T. Coffey, Yu P. Kalmykov and J. T. Waldron, The Langevin Equation: With Applications in Physics, Chemistry and Electrical Engineeing (World Scientific, 1996).

12. R. Ritz and T. J. Sejnowski, Curr. Opin. Neurobiol. 7 (1997) 536.

13. B. Schäfer, M. G. Rosenblum and J. Kurths, Nature (London) 392 (1998) 239.

14. B. Blasius, A. Huppert and L. Stone, Nature (London) 399 (1999) 354.

15. J. A. Freund, L. Schimansky-Geier and P. Hänggi, Chaos 13 (2003) 225.

16. J. D. Murray, Mathematical Biology (Springer-Verlag, 1993).

17. F. C. Hoppensteadt, Analysis and Simulation of Chaotic Systems, Applied Mathematical Sciences (Springer-Verlag, 1993).

18. D. Zwillinger, Handbook of Differential Equations (Academic Press, 1992).

19. H. U. Voss, Phys. Rev. E61 (2000) 5115.

20. O. Calvo, D. R. Chialvo, V. M. Eguiluz, C. Mirasso and R. Toral, Chaos 14 (2004) 1.

21. C. Mirasso, E. Hernandez-Garcia and C. Masoller, Phys. Lett. A295 (2002) 39.

22. H. U. Voss, Phys. Rev. Lett. 87 (2001) 014102.

23. C. Masoller, Phys. Rev. Lett. 86 (2001) 2782.

24. H. U. Voss, Phys. Lett. A279 (2001) 207.

25. H. Voss, Int. J. Bifurc. Chaos 12 (2002) 1619.

26. P. Davis, T. Aida, S. Saito, Y. Liu, Y. Takiguchi and J. M. Liu, Appl. Phys. Lett. 80 (2002) 4306.

27. E. M. Izhikevich, Int. J. Bifurc. Chaos 10 (2000) 1171.

28. A. L. Hodgkin and A. F. Huxley, J. Physiol. (London) 117 (1952) 500.

29. R. FitzHugh, Biophys. J. 1 (1961) 445.

30. J. S. Nagumo, S. Arimoto and S. Yoshizawa, Proc. IRE 50 (1962) 2061.

31. A. A. Andronov, E. A. Leontovich, I. I. Gordon and A. G. Maier, Qualitative Theory of Second-Order Dynamic Systems (Wiley, New York, 1973).

32. P. Coullet, T. Frisch, J. M. Gilli and S. Rica, Chaos 4 (1994) 485.

33. E. A. Jackson, Perspectives of Nonlinear Dynamics, Vol. 1 (Cambridge University Press, 1989).

34. J. Marsden and M. MacCracken, The Hopf Bifurcation and its Applications (SpringerVerlag, 1976).

35. A. L. Hodgkin, J. Physiol. 107 (1948) 165.

36. M. Ciszak, O. Calvo, C. Massoler, C. Mirasso and R. Toral, Phys. Rev. Lett. 90 (2003) 204102.

37. R. Toral, C. Massoler, C. Mirasso, M. Ciszak and O. Calvo, Physica A325 (2003) 192. 
38. M. Ciszak, F. Marino, R. Toral and S. Balle, Phys. Rev. Lett. 93 (2004) 114102.

39. P. König, W. Singer, A. K. Engel and A. K. Kreiter, Proc. Natl. Acad. Sci. USA 88 (1991) 6048.

40. P. König, W. Singer, P. R. Roelfsema and A. K. Engel, Nature 385 (1997) 157.

41. W. J. Freeman, Int. J. Bifurc. Chaos 10 (2000) 2307.

42. I. M. Stanford, J. G. R. Jefferys, R. D. Traub and M. A. Whittington, Nature 383 (1996) 421.

43. J. M. Barrie and W. J. Freeman, J. Neurophysiol. 84 (2000) 1266.

44. Ch. Koch and F. Crick, Sci. Am. 12 (August 2002), p. 11.

45. V. Dragoi, J. Sharma and M. Sur, IETE J. Research 49 (2003) 2. 